\title{
SPECTRAL GRAPH WAVELET BASED NONRIGID IMAGE REGISTRATION
}

\author{
Nhung Pham, David Helbert, Pascal Bourdon, Philippe Carré \\ XLIM/ASALI, UMR CNRS 7252, University of Poitiers \\ BP 30179, 86962 Futuroscope-Chasseneuil Cedex, France. \\ Email: hong.nhung.pham@univ-poitiers.fr
}

\begin{abstract}
We propose a nonrigid registration method whose motion estimation is cast into a feature matching problem under the Log-Demons framework using Graph Wavelets. We investigate the Spectral Graph Wavelets (SGWs) to capture the shape feature of the images. The advantages of the Spectral Graph Wavelet (SGW) over the classical wavelet is that data representation on graphs is more adapted to data with complex structures. Our experiments on T1 brain images and endomicroscopic images show that this method outperforms the existing nonrigid image registration techniques (i.e. LogDemons and Spectral Log-Demons) with improved similarity values.
\end{abstract}

Index Terms - Image Registration, nonrigid deformation, Graph Wavelets, Log-Demons Registration

\section{INTRODUCTION}

Nonrigid image registration is critical for many applications in computer vision such as medical image applications, atlas construction and remote sensing applications. Nonrigid methods can be classified into two categories: intensity based [1, 2], and geometric based [3, 4]. The former finds correspondence between images using intensity values while the latter deals with the image structures. Intensity based method mostly relies on a gradient based method to optimize the intensity difference between images, which suffers from a various number of aspects (e.g. local minima, noise, image distortion). In contrast, the geometric based method is more robust to structure changes and scene movements with global transformation but limited in case of local deformation. Motivated by the fact that intensity based and geometric based methods complement each other, many applications have combined these two techniques for large and complex deformations [5, 6], and they have facilitated many other studies.

Thanks to the development of computer science, the idea of applying graph theory in image processing has been extensively explored. This is due to its simplicity to provide a discrete and mathematical representation of different types

The authors would like to thank Lombaert et al and Hammond et al for making their source codes available online. of images. It was observed in [5] that the graph spectrum is geometric invariant. This work obtained a noticeable success in diffeomorphic registration with large and complex deformation that utilizes graph spectrum in the Log-Demons framework. However, experiments showed that the graph spectrum is noise prone.

On the other hand, one of the widely used geometric based approaches is wavelet based registration because of its ability to perform multiresolution scheme and image representation in both time and frequency domains, as well as its ability to characterize texture images. In this case, the image features are extracted via wavelet coefficient. The registration step then becomes a feature matching problem [4, 7, 8, 9]. Although wavelet decomposition preserves strong features, these methods are limited to rigid and affine transformations.

Recent research on graph theory is proposed by Hammond et al [10]. They introduced a new computation of Spectral Graph Wavelet Transform that is defined in the graph Fourier domain. This allows us to utilize the benefits of the image representation on graph while keeping the advantages of wavelets. The advantages of wavelets are the image representation at different frequencies and the ability to accentuate shape feature. A prominent number of recent SGW based applications in computer vision, such as shape classification [11], shape retrieval [12, 13], local texture characterization[14], image denoising [15] have proven the benefits of Graph Wavelets.

In this paper, we propose a SGW based nonrigid image registration method. We investigate the lower bands of the SGWs on entire image at different scales as the geometric constraint of the similarity measurement, together with the intensity and spatial constraints. In order to archive diffeomorphic registration, the motion estimation is under the well known Log-Demons framework. To the best of our knowledge, this is the first time SGW is applied to the problem of nonrigid image registration. Our experiments show that our method outperforms the existing methods, especially when noise exists. 


\section{THEORY AND METHOD}

\subsection{Non-parametric Image Registration}

Classically, image registration is the process of spatially aligning a source image $I_{S}$ to fit a target image $I_{T}$. The goal of non-parametric registration is to find the transformation $s(p)$ from $I_{T}$ to $I_{S}$ for each point $p$, such that for the full field $I_{S \circ s}=I_{T}$. The transformation is obtained by optimizing the energy function that contains intensity similarity and regularization energy:

$$
E\left(I_{T}, I_{S}, s\right)=\alpha_{i} E_{s i m}\left(I_{T}, I_{S \circ s}\right)+\alpha_{r} E_{r e g}(s),
$$

where $\alpha_{i}$, and $\alpha_{r}$ represent the intensity and regularization weights of registration. The intensity and regularization energy forms are denoted by the sum of square differences of image intensity and spatial coordinates: $E_{\text {sim }}\left(I_{T}, I_{S \circ s}\right)=$ $\left\|I_{T}-I_{S \circ s}\right\|^{2}$, and $E_{r e g}(s)=\left\|X_{T}-X_{S \circ s}\right\|^{2}$, with $X_{T}, X_{S}$ are spatial coordinates of target and source images.

\subsection{Data Representation by Graph}

Graph definition: A graph is defined as $G=(\mathcal{V}, \mathcal{E})$, where $\left.\mathcal{V}=v_{0}, \ldots, v_{N-1}\right\}$ is a set of vertices and $\mathcal{E}$ is a set of edges representing the weight between vertices [16]. In graph based image processing, vertices can represent pixels and therefore, edges represent the neighboring structure of these pixels. Such graph can be represented on an adjacency matrix $A$ where $A_{i j}$ is the undirected edge weight of vertex $v_{i}$ and vertex $v_{j}$.

Graph Laplacian Matrix: Matrix $D$ is a diagonal degree matrix where each value $D_{i i}$ on the diagonal is the sum of the weights of all the edges incident to vertex $v_{i}$, such that: $D_{i i}=\sum_{j} A_{i, j}$. The Laplacian matrix is given as: $L=D-A$. The normalized Laplacian matrix introduced in [16] is given as: $\mathcal{L}=D^{-1 / 2} L D^{-1 / 2}$.

Obviously, graph construction is about how Laplacian matrix is built. It fully depends on how points are connected and the computation of the edge weights. This will be briefly discussed later in this paper.

\subsection{Spectral Graph Wavelet Transform}

Graph Fourier Transform: Given graph signal $f \in \mathbb{R}^{N}$, according to [17] the graph Fourier transform of the signal is defined as:

$$
\hat{f}(\ell)=<\mathrm{v}_{\ell}, f>=\sum_{n=1}^{N} \mathrm{v}_{\ell}^{*}(n) f(n),
$$

where $\left\{\lambda_{\ell}, \mathrm{v}_{\ell}\right\}_{\ell=1, \ldots, n}$ forms eigensystem of Laplacian matrix, with eigenvalues $0 \leq \lambda_{1} \leq \lambda_{2} \leq \cdots \leq \lambda_{N}=\lambda_{\max }$, and associated eigenvectors $\mathrm{v}_{1}, \mathrm{v}_{2}, \ldots, \mathrm{v}_{N}$.

Graph wavelet transform: The SGWs are modulated by a generating kernel $g$, which performs as a band-pass filter in the spectral domain, $T_{g}^{t}$ denotes the wavelet operators at scale $t$.

Wavelet coefficients of graph signal $f$ at scale $t$ are defined as:

$$
W_{f}(t, i)=<\psi_{t, i}, f>=\sum_{\ell=1}^{N} g\left(t \lambda_{\ell}\right) \hat{f}(\ell) \mathrm{v}_{\ell}(i) .
$$

In order to encode the low frequency of signal $f$, a second class of waveforms $h$ is defined similarly to the low pass filter in classical wavelet transform, such that: $h(0)>0$ and $\lim _{x \rightarrow \infty} h(x)=0$. This scaling function $S_{f}(n)$ is denoted by:

$$
S_{f}(i)=(h(t \mathcal{L}) f)(i)=\sum_{\ell=1}^{N} h\left(\lambda_{\ell}\right) \hat{f}(\ell) \mathrm{v}_{\ell}(i) .
$$

\section{PROPOSED REGISTRATION ALGORITHM}

\subsection{Graph Computation}

The construction of a graph mainly focuses on point connectivity and the computation of the edge weights. The Simplest definition of adjacency matrix can be uniform, in which $A_{i j}=1$ if $v_{i}, v_{j}$ are connected and $A_{i j}=1$ otherwise. In a more advanced way, edge weight can rely on the intensity difference between vertices, given as below:

$$
A_{i j}= \begin{cases}\exp \left(-\frac{\left(I_{i}-I_{j}\right)^{2}}{K^{2}}\right) & \text { if }(i, j) \in \mathcal{E}, \\ 0 & \text { otherwise }\end{cases}
$$

Parameter $K>0$ controls the sensitivity to edges in the images. In another word, the value of $K$ affects the influence of the difference between every two adjacent pixels. When the value of $K$ increases, the influence of pixel difference decreases. It is chosen depending on the noise and texture characteristics of the image.

Concerning the graph topology, in fact, there is no general rule of how points are connected. In general case, one can use the k-nearest neighbor scheme to find adjacent points, or for each point, choose its adjacent points in one circle. In case of regular data, such as image, each pixel in the image is considered as one vertex, each vertex can connect with its four closest neighbors, or with additional diagonal neighbors. In principle, for stronger graph connection, edges can be built on pixels with any larger spatial distance, but the graph communication cost will increase.

\subsection{Objective function}

At this stage, we briefly introduced the graph representation and construction. We also described the SGW decomposition. To recall to the problem of the state-of-art, classical intensity based methods are limited to the local scope and lacks 
geometric invariance. A spectral approach that is geometric invariant is well known to adapt with non-local deformation. Such complementary approaches motivate a hybrid method that combines intensity based and geometric based. SGW approach is a multiscale graph based technique, that is defined in graph spectral Fourier domain. Therefore, SGW not only is geometric invariant but also allows us to analyze graph data in different frequencies. In this section, we provide our objective function that contains SGW constraint for geometric invariance. Suppose we analyze the SGWs of each image at $t$ scales, the constructed Graph Wavelets then compose of $t+1$ components, where the first component is of the scaling function, and the rest are the SGWs at scales $1, \ldots, t$. Each component is at the same size with the image. We choose the first $k$ components to use for registration, denoted as $W_{T}$ and $W_{S}$ for the target and source images. The choice of $t$ and $k$ depends on the image characteristics. In fact, $t$ controls how image details are stretched in frequency domain, and $t$ defines the levels of details that we want to use.

We exploit the specifications of the generating kernel function $g$ and the scaling function $h$ in [10]. Our objective function is defined as:

$$
\begin{array}{r}
E\left(I_{T}, I_{S}, s\right)=\alpha_{i}\left\|I_{T}-I_{S \circ s}\right\|^{2}+\alpha_{r}\left\|X_{T}-X_{S \circ s}\right\|^{2} \\
+\alpha_{w}\left\|W_{T}-W_{S \circ s}\right\|^{2},
\end{array}
$$

where $\alpha_{i}, \alpha_{r}$, and $\alpha_{w}$ are respectively the intensity, regularization, and spectral wavelet weights. In each iteration, the transformation field is achieved by performing optimization on Lie algebra [?] through an exponential map of the stationary velocity $v$ according to [1]. SGWs are computed for both updated images. The update fields $u_{f w}$ and $u_{b w}$ are obtained by a nearest neighbor search, such that the energy defined in Eq. 6is minimized in both forward $\left(I_{T}\right.$ to $\left.I_{S \circ s}\right)$ and backward ( $I_{S}$ to $\left.I_{T \circ s^{-1}}\right)$ matching. The symmetric update field $u$ is the average of $u_{f w}$ and $-u_{b w}: \frac{1}{2}\left(u_{f w}-u_{b w}\right)$. The next step is to smooth the update field $u$ with a Gaussian kernel $K_{\text {fluid }}$ with standard deviation $\sigma_{\text {fluid }}$. The velocity field $v$ is updated by the computed update field, and then smoothed with a Gaussian kernel $K_{\text {diff }}$ with $\sigma_{\text {diff }}$. The registration process can be summarized as in Algorithm 1 .

\section{RESULTS}

In order to show the benefits of our proposal, we benchmark our method against two existing algorithms (i.e. Log-Demons and Spectral Log-Demons). We use the same set of weighting parameters $\left(\alpha_{i}=20, \alpha_{r}=4, \alpha_{w}=1 . \alpha_{w}\right.$ is the spectral weight of Spectral Log-Demons, and SGW weight in our method). We use eight neighbor graph connectivity in all of the experiments. To have an application that is less sensitive to noise, we choose a large value of $K(K=30)$ to have less influence of intensity difference on the computation of edge weight (Eq. 5). We use $t=4$ number of scales for SGW com-

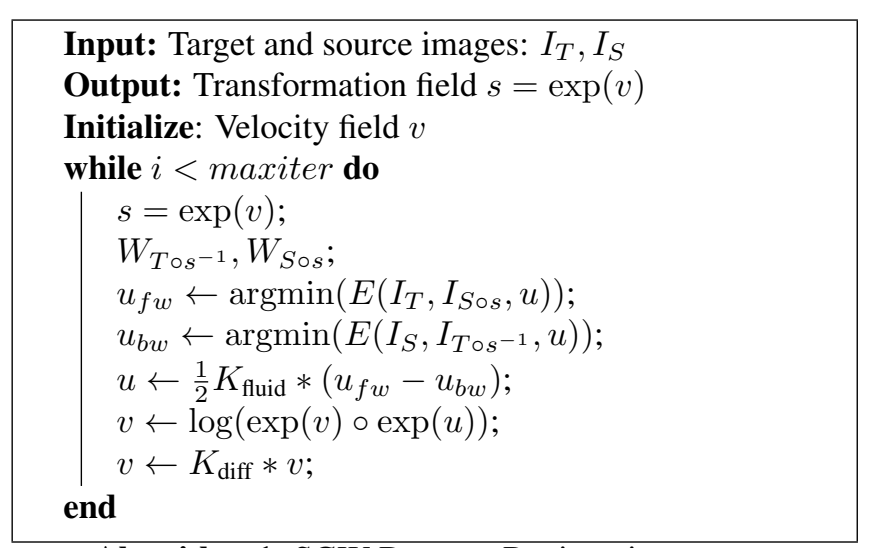

\section{Algorithm 1: SGW Demons Registration}

putation and choose $k=3$ first components at lower bands (including the scaling function component) for registration.

Fig. 1 shows the output of the proposed algorithm compared to existing solutions. The first and second rows of show the registration results on two $\mathrm{T} 1$ images. In the first row, the target and source images are shown on the first and second images, the following from left to right are the registered images of the Log-Demons, Spectral Log-Demons and our method respectively. The second row displays the corresponding graphical Absolute Difference of the methods. The top right figure of Fig. 1 shows the result in term of Mean Square Error (MSE) between the registered image and the target image of all algorithms with respect to the iteration step. Similarly, the third and fourth rows show the registration results on endomicroscopic images. The target image is cropped from an endomicroscopic image. The source image is generated by performing a random bspline transform on the target image. The bottom right figure shows the MSE curves of the methods with respect to number of iterations.

As can be seen in Fig. 1, the methods with a geometric constraint (Spectral Log-Demons method and our method) fall faster to the convergence of the registration. However, in the registration of T1 images, at further iterations, the MSE of the Log-Demons method decreases and gets close to the MSE value of the Spectral Log-Demons method.

In order to show that the SGW based method is robust to noise, we add a Gaussian noise to the target and source images with standard deviation $\sigma_{\text {noise }}$ ranging from 0 to 0.1 . The MSE value of the algorithms with respect to noise is shown in Fig. 2. To see more clearly, we shows the target (in red channel) and registered (in green channel) images of the algorithms when $\sigma_{\text {noise }}=0.014$ at the rightmost of the figure. The significant misalignment of Log-Demons and Spectral Log-Demons are shown in the cropped regions (in blue rectangle close to the nose, and in yellow rectangle close to the neck region). We observe that the performance of the Spectral Log-Demons method fluctuates unpredictably with respect to noise. But in all of the tested cases, our proposal provides the 

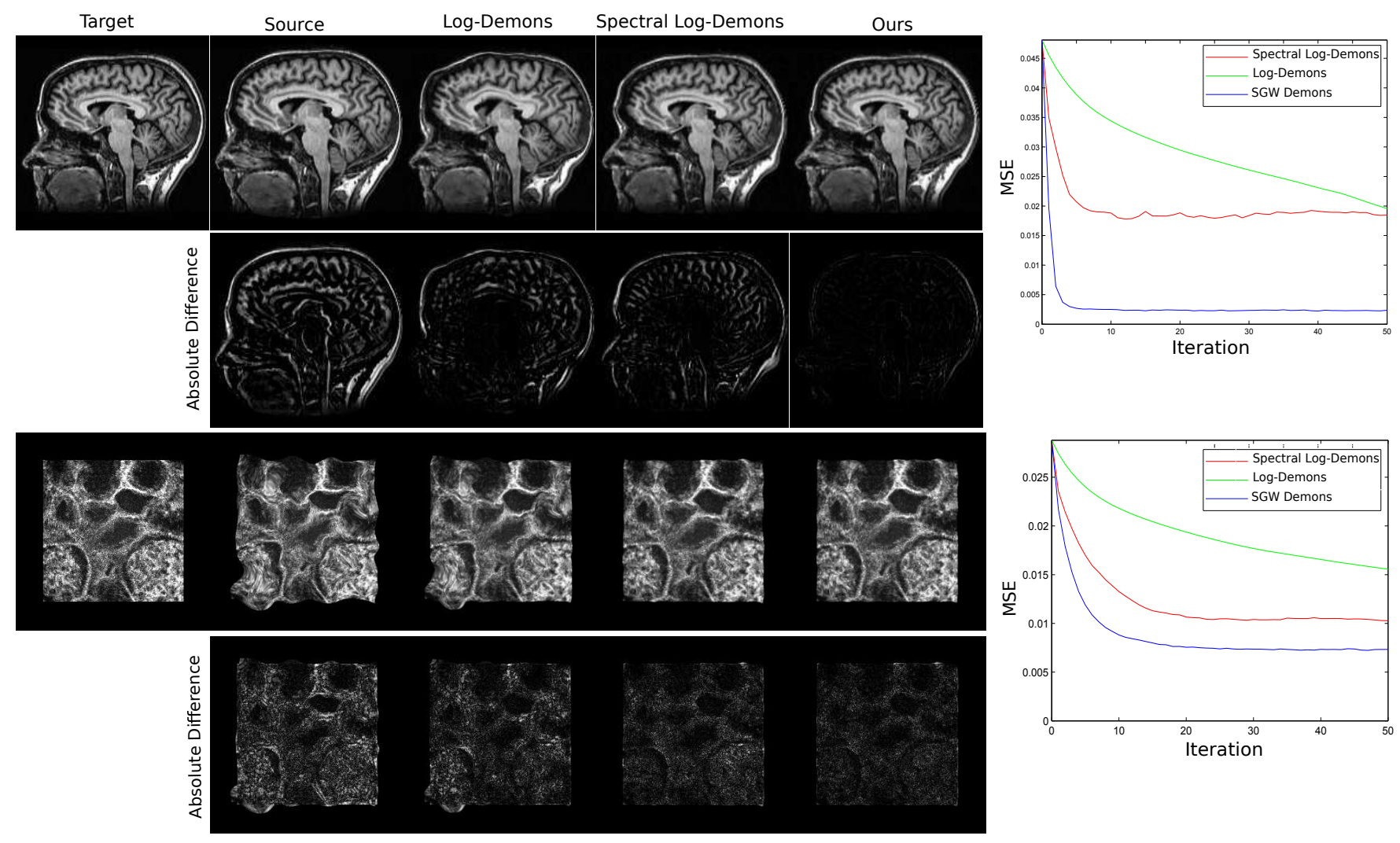

Fig. 1. Comparison between our proposal and the existing methods when no noise is added

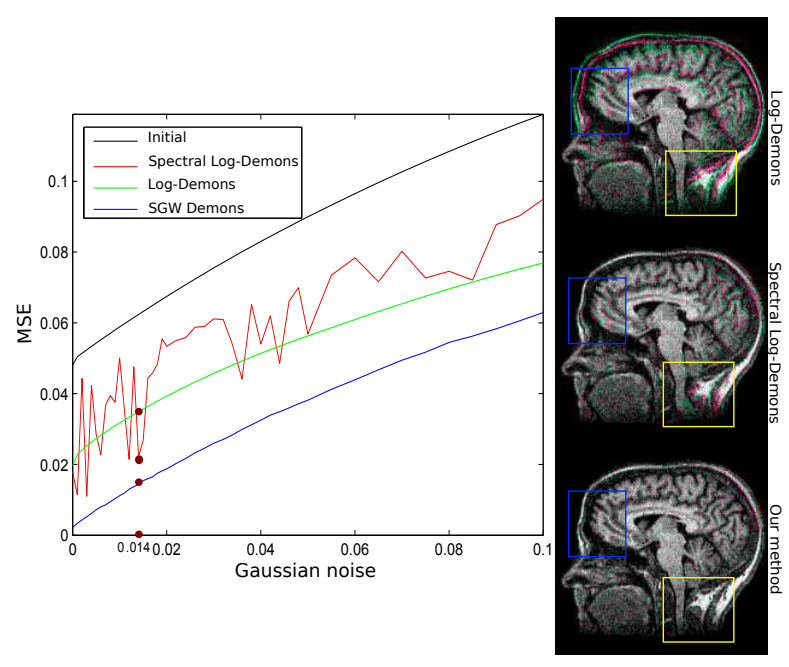

Fig. 2. MSE of Log-Demons, Spectral Log-Demons methods, and our method with respect to Gaussian noise (standard deviation $\sigma_{\text {noise }}$ )

best results compared to other methods.

\section{CONCLUSIONS AND FUTURE WORKS}

In this paper, we proposed a nonrigid image registration method based on a hybrid approach that consists of intensity based and SGW based matching. Our contribution is first, we showed that the SGWs can be applied to spectral matching, and then adapted to the Log-Demons framework. Secondly, the SGWs that can represent strong shape features can be applied to noisy images. Our experimental results on brain images at different levels of Gaussian noise show that our method outperforms the existing methods on a similar framework, and more robust to noise. However, graph based methods are still limited to low resolution images. In our future work, we will experiment on different graph connectivities and the computation of edge weights to study how they affect the image registration.

\section{REFERENCES}

[1] T. Vercauteren, X. Pennec, A. Perchant, and N. Ayache, "Diffeomorphic demons: Efficient non-parametric image registration," NeuroImage, vol. 45, no. 1, pp. S61S72, 2009.

[2] B. D. Lucas, T. Kanade et al., "An iterative image reg- 
istration technique with an application to stereo vision." in IJCAI, vol. 81, no. 1, 1981, pp. 674-679.

[3] P. Bourdon and D. Helbert, "A data-driven approach to feature space selection for robust micro-endoscopic image reconstruction," in IEEE International Conference on Image Processing (ICIP), 2017.

[4] G. Hong and Y. Zhang, "Wavelet-based image registration technique for high-resolution remote sensing images," Computers \& Geosciences, vol. 34, no. 12, pp. 1708-1720, 2008.

[5] H. Lombaert, L. Grady, X. Pennec, N. Ayache, and F. Cheriet, "Spectral demons-image registration via global spectral correspondence," in Computer VisionECCV 2012. Springer, 2012, pp. 30-44.

[6] K. C. Lam and L. M. Lui, "Landmark-and intensitybased registration with large deformations via quasiconformal maps," SIAM Journal on Imaging Sciences, vol. 7, no. 4, pp. 2364-2392, 2014.

[7] L. M. Fonseca and M. H. Costa, "Automatic registration of satellite images," in Computer Graphics and Image Processing, 1997. Proceedings., X Brazilian Symposium on. IEEE, 1997, pp. 219-226.

[8] H. Xishan and C. Zhe, "A wavelet-based multisensor image registration algorithm," in Signal Processing, 2002 6th International Conference on, vol. 1. Ieee, 2002, pp. 773-776.

[9] C. Paulson, S. Ezekiel, and D. Wu, "Wavelet-based image registration," in Evolutionary and Bio-Inspired Computation: Theory and Applications IV, vol. 7704. International Society for Optics and Photonics, 2010, p. 77040M.

[10] D. K. Hammond, P. Vandergheynst, and R. Gribonval, "Wavelets on graphs via spectral graph theory," Applied and Computational Harmonic Analysis, vol. 30, no. 2, pp. 129-150, 2011.

[11] M. Masoumi and A. B. Hamza, "Shape classification using spectral graph wavelets," Applied Intelligence, vol. 47, no. 4, pp. 1256-1269, 2017.

[12] M. Masoumi, C. Li, and A. B. Hamza, "A spectral graph wavelet approach for nonrigid 3d shape retrieval," Pattern Recognition Letters, vol. 83, pp. 339-348, 2016.

[13] C. Li and A. B. Hamza, "A multiresolution descriptor for deformable 3d shape retrieval," The Visual Computer, vol. 29, no. 6-8, pp. 513-524, 2013.

[14] M. T. Pham, G. Mercier, and J. Michel, “Textural features from wavelets on graphs for very high resolution panchromatic pléiades image classification," Revue française de photogrammétrie et de télédétection, vol. 208, pp. 131-136, 2014.

[15] M. Malek, D. Helbert, and P. Carré, "Color graph based wavelet transform with perceptual information," Journal of Electronic Imaging, vol. 24, no. 5, p. 053004, 2015.

[16] F. R. Chung, Spectral graph theory. American Mathematical Soc., 1997, vol. 92.

[17] A. Sandryhaila and J. M. Moura, "Discrete signal processing on graphs," IEEE transactions on signal processing, vol. 61, no. 7, pp. 1644-1656, 2013. 